\title{
Processing of affective pictures modulates right-hemispheric gamma band EEG activity
}

\author{
Matthias M. Müller*, Andreas Keil, Thomas Gruber, Thomas Elbert \\ Department of Psychology, University of Konstanz, Fach D25, D-78457 Konstanz, Germany
}

\begin{abstract}
The present study was designed to test differential hemispheric activation induced by emotional stimuli in the gamma band range (30-90 $\mathrm{Hz}$ ). Subjects viewed slides with differing emotional content (from the International Affective Picture System). A significant valence by hemisphere interaction emerged in the gamma band from 30-50 Hz. Other bands, including alpha and beta, did not show such an interaction. Previous hypotheses suggested that the left hemisphere is more involved in positive affective processing as compared to the right hemisphere, while the latter dominates during negative emotions. Contrary to this expectation, the $30-50 \mathrm{~Hz}$ band showed relatively more power for negative valence over the left temporal region as compared to the right and a laterality shift towards the right hemisphere for positive valence. In addition, emotional processing enhanced gamma band power at right frontal electrodes regardless of the particular valence as compared to processing neutral pictures. The extended distribution of specific activity in the gamma band may be the signature of cell assemblies with members in limbic, temporal and frontal neocortical structures that differ in spatial distribution depending on the particular type of emotional processing.
\end{abstract}

Keywords: Emotion; Valence; EEG; Gamma band; Hemisphere asymmetry

\section{Introduction}

The investigation of brain asymmetries for cognitive functioning has a long history in neuropsychology. Evidence has been obtained in healthy subjects and also in patients with various brain lesions. Generally, left hemisphere cognitive strategies are described as detailed, analytic, and involving abstract logical reasoning, while right hemisphere strategies are thought to be configurational, synthetic, spatial, and related to more concrete perceptual insight (see Davidson and Hugdahl, 1995; Hellige, 1993 for an extensive overview). Brain asymmetries for emotional processing are controversial (reviewed in Borod, 1992). In general, the valence hypothesis predicts right hemisphere dominance for negative and lateralization towards the left hemisphere for processing of positive emotional material (e.g. Tucker, 1981; Silberman and Weingartner, 1986). However, an important distinction in neuropsychological research on emotion is made on the expression/experience or perception of emotions. Consequently, two versions of the valence hypothesis exist. In addition to the above

* Corresponding author. Tel.: +49-7531-882-696; fax: +49-7531-882891.

E-mail address: matthias.m.mueller@uni-konstanz.de (M.M. Müller) mentioned version, the second version hypothesizes a difference in hemispheric specialization with respect to expression or experience of emotion only, whereas the right hemisphere is dominant for the perception of emotions, regardless of valence (e.g. Hirschman and Safer, 1982; Davidson, 1984; Ehrlichman, 1987).

But also intrahemispheric differences have been proposed. Davidson (1984, 1995); Davidson et al. (1990). Fox (1991) have suggested a model in which withdraw behavior is linked to anterior regions of the right hemisphere and approach behavior to anterior regions of the left hemisphere (Davidson, 1984, 1995). In addition, the expression and experience of emotions is linked to anterior regions which are related to affect and the perception of emotions is hypothesized as a more cognitive function, linked to posterior regions (Davidson, 1984). A possible explanation for these hemispheric asymmetries could be given by linking these features to asymmetric cortico-cortico or corticolimbic emotional networks (Ledoux, 1995). Derryberry and Tucker (1992) discussed ascending and descending connections between brainstem, limbic, paralimbic and neocortical regions and suggested more pronounced connections between these subcortical structures and neocortex in the right hemisphere. One line of evidence, according to the authors, is their finding of higher coherence in right central 
and posterior regions during sexual arousal induced by imagination (Tucker and Dawson, 1984). According to Liotti and Tucker (1995), the right hemisphere is more specialized for the information representation of the dorsal pathway, which is spatial orienting, whereas the left hemisphere is dominated by the object recognition of the ventral pathway. Different limbic connections to the respective pathway give rise to the emotional processing and/or interpretation of stimuli resulting in the above mentioned asymmetries. Although this model is highly speculative and there is no evidence for more and different anatomical connections between the right hemisphere and subcortical or limbic structures (Borod, 1992), the model makes an important point. Emotions are represented in cortico-limbic networks rather than in particular areas of the brain. During processing of emotional material, such a network produces wide spread, rather than focal cortical activity.

High-frequency neural oscillations have been proposed as the physiological signature of associative networks or Hebbian cell assemblies. By synchronizing their firing patterns, neurons in a cell assembly would produce EEG activity in the gamma band when the assembly is ignited (Eckhorn et al., 1990; Singer and Gray, 1995; Pulvermüller et al., 1997). In humans, non-invasive electro- (EEG) and magnetoencephalographic (MEG) recordings have provided evidence that cortical activity in the gamma band range (above $20 \mathrm{~Hz}$ ) is modulated by (a) the features of a stimulus (Müller et al., 1996; Tallon et al., 1995; Müller et al., 1997; Tallon-Baudry et al., 1997b), by (b) visual spatial attention (Müller, 1998; Gruber et al., 1999), and (c) may represent the perception of a Gestalt (Tallon-Baudry et al., 1997a,b; Keil et al., 1999). In addition, power in the gamma band was enhanced during the active representation of an object in short-term memory during the delay of a delayed-matching-to-sample task (Tallon-Baudry et al., 1998). Since this activity was found at occipito-temporal and frontal leads, the authors interpreted the finding as supportive for the idea of a synchronized cortical network involving prefrontal and ventral visual areas.

In a similar manner, Pulvermüller et al. (1995) and Pulver müller et al. (1997) have proposed a model in which Hebbian cell assemblies are the key mechanisms for language processing. Different topographies of cortical gamma band activity were found with words eliciting either motor (more over central areas) or visual associations (more over occipital areas). The author concludes that an action verb like 'to catch' is represented in a cell assembly which encloses neurons in perisylvian and motor cortices (Pulvermüller et al., 1995). In sum, experiments in humans indicate that cortical activity in the gamma band range is related to visual and language information processing, object representation, and possibly to feature integration. It seems plausible that this activity is an index of high frequency synchronization of wide spread cortical cell assemblies. Thus, there is converging evidence that high frequency power in the gamma range of the human EEG or MEG seems not to be an index of cortical desynchronization as commonly interpreted for the beta band, but rather should be seen as an index of neural synchronization of forming cortical networks (Müller, 1998).

If emotions are represented in distributed cell assemblies with synchronized neural high frequency firing, the valence hypothesis in its main version would predict that positive valence is related to higher gamma band activity over the left and negative valence to higher gamma band activity over the right hemisphere. In a statistical analysis of variance, this should result in a significant hemisphere by valence interaction. As of today, research on cortical emotional responses in the frequency domain was mainly focused on alpha desynchronization (see Davidson, 1995, for an overview). Alpha desynchronization, however, is a poor predictor for measuring the activity of cortical networks since topographical distributions of alpha and high frequency responses are not inversely related in an one-by-one fashion (Müller et al., 1996; Müller et al., 1997; Müller, 1998; Tallon-Baudry et al., 1998). Ray and Cole (1985), for instance reported higher beta activity (16$24 \mathrm{~Hz}$ ) in right hemisphere temporal and parietal areas, whereas no effect was found in the alpha band to reflect emotional processes. Furthermore, Aftanas and co-workers (1998) have found that their measurement for non-linear dynamical coupling of different brain areas distinguished between positive and negative valence for higher frequencies (up to $28 \mathrm{~Hz}$ ) but not for the alpha band. Thus, it seems interesting to test high frequency responses for their sensitivity to the processing of emotional stimuli and the experience and expression of emotions.

Previous research in the field has focussed on the alpha band during imagination or presentation of video clips. Alpha activity of positive valence was compared to that of negative valence. Most of the above-cited studies did not correct their data for both general noise (i.e. baseline) and the effect of simply watching visual stimuli. Consequently, we compared the effects of positive and negative valence by correcting the power of a respective frequency band by the power while the subject was observing neutral pictures. A further limitation of previous research is the small number of electrodes used. In most cases only 6-8 electrodes on left and right frontal, central, parietal and - very rarely - occipital sites were recorded (see Tucker and Dawson, 1984; Ray and Cole, 1985; Fox, 1991; Davidson, 1995). Such a limited number of electrodes is suboptimal for investigating cerebral hemispheric asymmetries and provides only restricted topographic evidence of cortical network activity. Here, we used a 128 channel high density recording for a near optimal documentation of the topography of cortical activity in specific frequency bands. Also, we used a large sample of validated emotional picture material, the International Affective Picture System (IAPS; Lang et al., 1997) to avoid habituation. In addition to the investigation of the valence hypothesis, we investigated for gamma band effects at frontal leads to test the anterior/posterior intrahemi- 
spheric hypothesis (Davidson, 1984; 1995; Davidson et al., 1990; Fox, 1991).

\section{Methods}

\subsection{Subjects}

Informed consent was received by 11 right-handed, male students with normal or corrected-to-normal vision (24-30 years, mean age of 26 years). Subjects were given class credits or a small financial bonus.

\subsection{Stimulation}

Sixty colored pictures were selected from the International Affective Picture System (Lang et al., 1997) and divided into 3 groups differing in affective valence: Twenty pictures depicted erotic couples or happy families, twenty pictures depicted emotionally neutral faces or household objects and a further 20 pictures depicted mutilated bodies or scenes associated with attack. The IAPS numbers of the pictures in the 3 groups are given in Appendix A. All pictures were presented in the center of a 19-inch computer screen with a frame rate of $75 \mathrm{~Hz}$. The screen was placed 1.5 $\mathrm{m}$ in front of the viewer, resulting in a picture presentation with a visual angle of 15 degrees horizontally and 11 degrees vertically. Each picture was presented for 6000 $\mathrm{ms}$, with interstimulus intervals varying between 7 and $12 \mathrm{~s}$.

\subsection{Procedure and electrophysiological recordings}

In a first training procedure, subjects were confronted with two examples of the affective stimuli for each affective category, respectively. In this training procedure subjects learned to maintain gaze on the center of the screen and to avoid explorative eye-movements. These pictures were not part of the experimental set. After that, the sensor net was applied and participants viewed two blocks with 60 pictures each, resulting in 40 trials per condition. In all blocks, the order of the stimuli was pseudo-randomized under the restriction that trains of three pictures with the same emotional valence should not occur. Subjects were instructed to avoid exploratory eye-movements and eyeblinks and attentively watch the pictures. After the EEG recordings, subjects viewed the 60 pictures again in a pseudo-randomized order and were asked to rate the respective picture on two categories, affective valence and arousal on the basis of a paper and pencil version of the self-assessment manikin (SAM; Bradley and Lang, 1994). The SAM consists of 3 scales, valence, arousal and dominance on a speech free scale ranging from 1 to 9 with higher values representing more positive valence (pleasureness), higher arousal and more feelings of dominance. As mentioned above, in the present experiment only the scales valence and arousal were used. SAM-ratings have been shown to be a reliable and valid indicator of an individual's emotional state (Bradley and Lang, 1994).

EEG was recorded continuously from 128 electrodes using an electrical geodesics system with $\mathrm{Cz}$ as reference. Impedance was kept below $50 \mathrm{k} \Omega$. All channels were preprocessed on-line by means of $0.1 \mathrm{~Hz}$ high-pass and $100 \mathrm{~Hz}$ low-pass filtering and digitized at a rate of 250 Hz. Further data processing was performed off-line.

\subsection{Data reduction and analysis}

For frequency analysis, single epochs of $7.000 \mathrm{~ms}$ length (1.000 ms before and $6.000 \mathrm{~ms}$ after picture onset) were extracted. These epochs were submitted for artifact rejection and correction using a procedure developed by Junghöfer et al. (statistical correction of artifacts in dense array studies, SCADS, unpublished data). This procedure (1) detects individual recording channel artifacts using the recording reference $(\mathrm{Cz}),(2)$ detects global artifacts using the average reference, (3) replaces sensors contaminated with artifacts throughout with statistically-weighted spherical interpolation from the full channel set, and (4) computes the variance of the signal across trials to document the stability of the averaged waveform. The limit for the number of approximated channels was set to 20 channels. Single epochs with excessive eye-movements and blinks and more than 20 channels containing artifacts were totally excluded from further analysis. This procedure led to the exclusion of two subjects due to excessive artifacts. For the remaining 9 subjects the mean rejection rate was $21 \%$. For further analysis, the averaged reference was used.

Since we were interested in the cortical activity which is not phase locked to the stimulus onset, the mean (i.e. event related potential, ERP) was subtracted from each single artifact free trial before transformation into the frequency domain by means of a fast Fourier transformation (FFT) approach similar to that described by Makeig (1993). This used the means of the FFT algorithm included in the AVG Q analysis software developed by Feige (1996). Each ERPcorrected epoch was subdivided into epochs of $768 \mathrm{~ms}$. One $768 \mathrm{~ms}$ epoch prior to picture onset, i.e. the dark screen, served as the baseline measure. After stimulus onset, 6 nonoverlapping $768 \mathrm{~ms}$ epochs were extracted and for all 7 epochs a Welch-tapered analysis window of $768 \mathrm{~ms}$ (192 sample points) was applied. The power spectrum for each analysis window was determined using two FFT windows containing 128 sample points $(512 \mathrm{~ms})$, with an overlap of 64 data points $(256 \mathrm{~ms})$, resulting in a frequency resolution of $2 \mathrm{~Hz}$. The relatively long analysis windows were chosen in order to compare low (alpha) and high (gamma) frequency bands on the basis of identical analysis windows. The resulting frequency matrices were averaged across all trials and the 6 analysis windows for each valence condition and electrode, respectively. This average across the 6 analysis windows was performed in order to increase the signalto-noise ratio for a given trial since each affective condition 


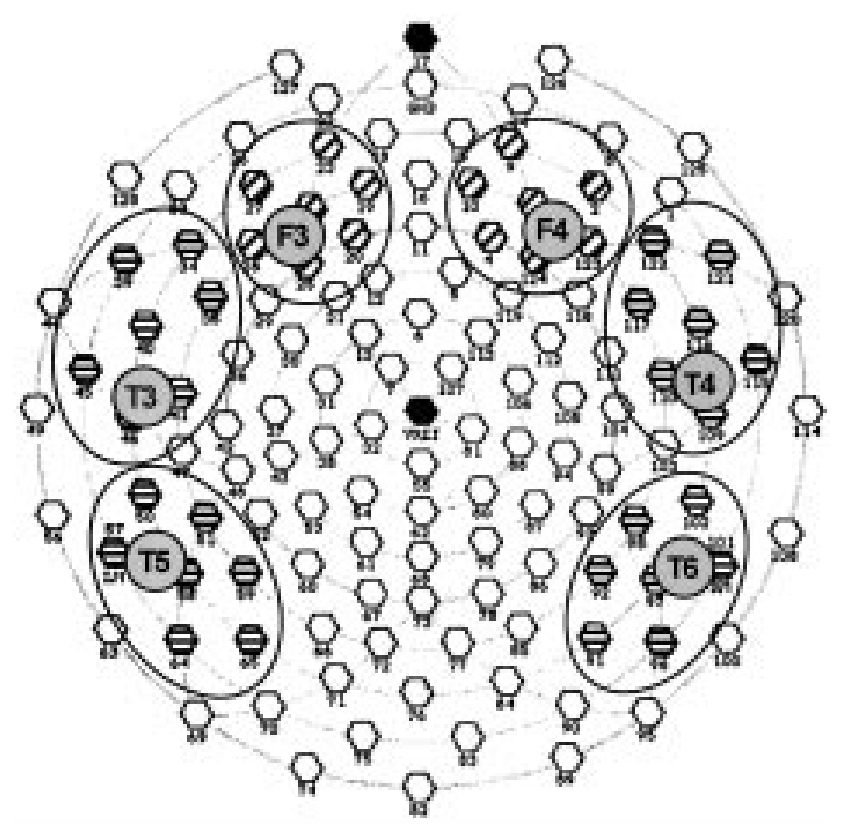

Fig. 1. Electrodes forming the frontal, temporal anterior and temporal posterior left and right hemisphere regional mean and its relative positioning to an electrode site of the international 10-20 system.

contained a maximum of 40 pictures only. The analysis window prior to picture onset was handled in the same way and subtracted from the respective mean of electrode and valence condition of each subject in order to correct for general noise. In order to obtain the 'pure' effects of positive or negative valence, corrected by effects of watching pictures, in the next step we subtracted the neutral from the pleasant and unpleasant conditions, respectively. Thus, results represent changes in a certain frequency band relative to the neutral condition.

\subsection{Statistical analysis}

For statistical analysis, 5 frequency bands were obtained by averaging across the respective frequencies: $\alpha=8-12$ $\mathrm{Hz}, \beta=13-19 \mathrm{~Hz}, \gamma-40=30-50 \mathrm{~Hz}, \gamma-60=50-70 \mathrm{~Hz}$, and $\gamma-80=70-90 \mathrm{~Hz}$. Temporal and frontal electrodes were clustered into regional means according to Fig. 1. Thus, each electrode cluster comprised seven electrodes. Fig. 1 displays the relative position of the selected clusters with respect to sites of the international 10-20 system.

Temporal sites were subject to a three factor repeatedmeasure analysis of variance (ANOVA; valence (pleasant/ unpleasant), hemisphere (left/right), and recording site (temporal anterior/temporal posterior)) for each frequency band, respectively. For frontal sites, a two factor repeatedmeasure ANOVA (valence (positive/negative), hemisphere (left/right)) was conducted for each frequency band.

SAM-ratings for valence and arousal were evaluated by means of one-factor repeated-measures ANOVAs (valence; pleasant/neutral/unpleasant) of the averaged ratings across pictures within each category. Where appropriate, degrees of freedom were adjusted by Greenhouse-Geisser epsilon. All post-hoc comparisons were evaluated by means of paired $t$ tests. One sample $t$ tests against zero were conducted where appropriate in order to test power changes as compared to the neutral condition or differences of SAMratings. Means and standard errors are presented.

\section{Results}

\subsection{SAM-ratings}

Fig. 2 shows the valence and arousal SAM-ratings for all 3 experimental conditions across subjects.

As expected, the statistical analysis revealed a highly significant effect for valence $(F(2,16)=461.4$, $P<0.001)$ and arousal $(F(2,16)=312.1, \quad P<0.001)$. For pleasant slides, overall SAM-ratings for valence were significantly higher as compared to neutral $(\mathrm{t}(8)=15.6$, $P<0.001)$ and unpleasant slides $(\mathrm{t}(8)=23.9$, $P<0.001)$. In addition, valence ratings for neutral slides were significantly higher as compared to unpleasant slides $(\mathrm{t}(8)=21.1, P<0.001)$. With respect to arousal ratings, arousal was rated significantly higher for pleasant as compared to neutral $(\mathrm{t}(8)=23.6, P<0.001)$, lower for pleasant as compared to unpleasant $(\mathrm{t}(8)=-6.6$, $P<0.001$ ), and lower for neutral as compared to unpleasant slides $(\mathrm{t}(8)=-21.4, P<0.001)$.

\subsection{Electrophysiological recordings}

The baseline corrected power across all 128 electrodes and 9 subjects are depicted in Fig. 3 for the frequencies in the gamma band range plus the mean power for the 3 gamma frequency bands, respectively.

As can be seen in Fig. 3, power in all three frequency bands for the neutral condition showed only a marginal increase as compared to the black screen. Power for positive and negative valence was most pronounced for $\gamma-40$.

Fig. 4 presents the grand mean spherical spline topographical distributions (Perrin et al., 1989) of baseline corrected alpha and $\gamma-40(30-50 \mathrm{~Hz})$ power for the left and right hemisphere for pleasant, neutral and unpleasant pictures. In addition, the back view for all three conditions is presented for the alpha band.

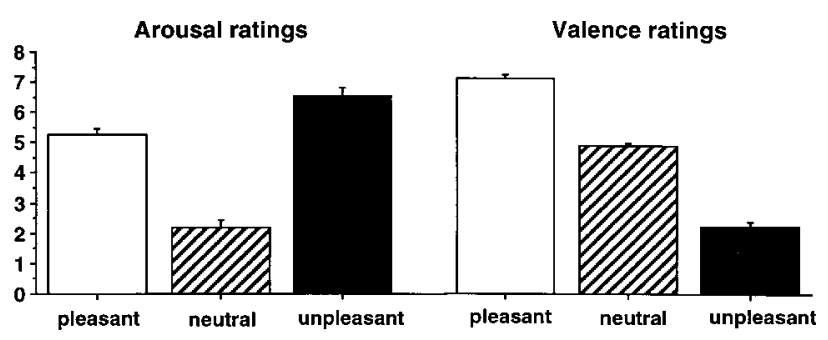

Fig. 2. Mean SAM arousal and valence ratings across subjects $(+$ standard error). 


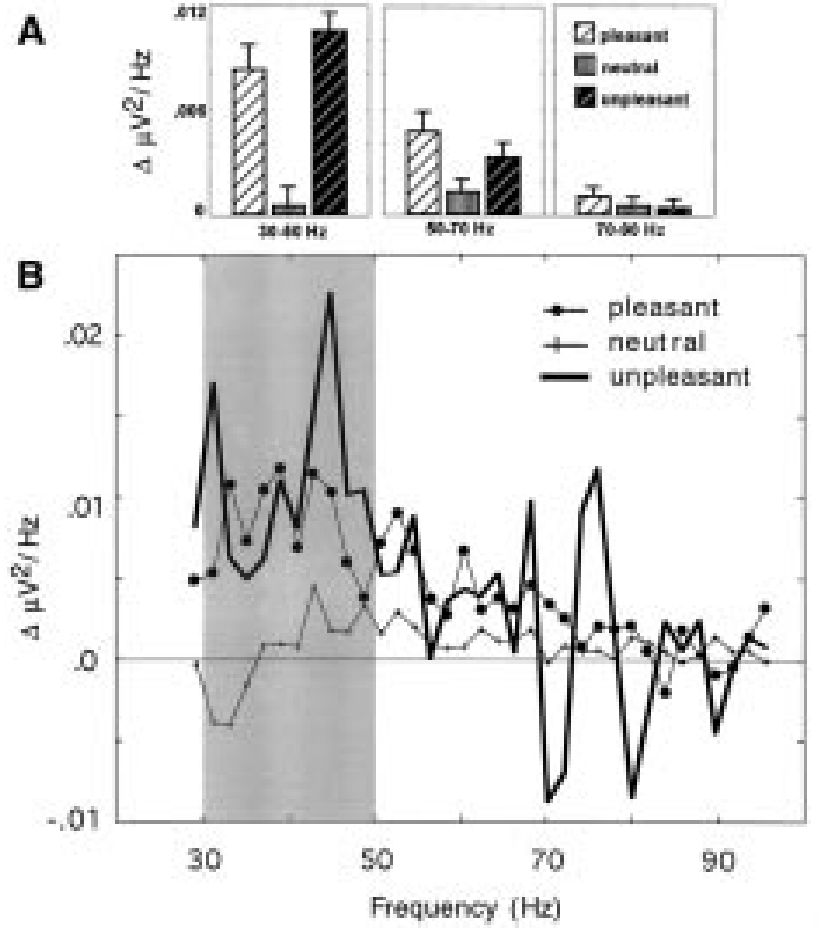

Fig. 3. Grand mean baseline corrected spectral power across 128 electrodes for the 3 affective categories (lower panel, B) and the resulting mean spectral power for the $30-50,50-70$ and $70-90 \mathrm{~Hz}$ frequency band (+ standard errors, upper panel, A).

As can be seen in Fig. 4 the center of baseline corrected activity in the $\gamma-40$ band for emotional pictures was over the anterior and posterior temporal lobe ventrally to electrodes $\mathrm{F} 3 / \mathrm{F} 4, \mathrm{C} 3 / \mathrm{C} 4$ and $\mathrm{P} 3 / 4$ of the international 10-20 system. In the neutral condition, the center of activity was more on parieto-occipital electrodes, indicating activity related to visual information processing. In comparison to the black screen, the back view of the alpha band clearly depicts a posterior alpha blocking when pictures were presented.

\subsection{Frontal electrode sites}

No statistically significant effect was found for alpha activity, indicating no sensitivity of alpha desynchronization with respect to emotional valence when corrected for the neutral condition. This was also true for all comparisons between pleasant and unpleasant and relative to the neutral condition. Beta difference activity was greater over the right hemisphere than over the left hemisphere for pictures with emotional content $(F(1,8)=17.9, P<0.003)$, whereas the left hemisphere exhibited a negative power, indicating more beta activity when processing neutral pictures. One sample $t$ tests against zero for the frontal right and left hemisphere beta power revealed no significant effect, indicating no statistically significant change in beta power activity as compared to processing neutral pictures.

With respect to $\gamma-40$ power corrected for the neutral condition, right frontal sites exhibited a significant increase in power for positive and negative valence relative to neutral stimuli $(\mathrm{t}(8)=3.6 ; 3.2, P<0.02)$. No effect could be detected with respect to $\gamma-60$ and $\gamma-80$ at the frontal leads.

\subsection{Temporal electrode sites}

With respect to alpha and beta, no statistically significant effect was found in the ANOVA model. In addition, no effect was present when comparing positive and negative valence to watching neutral pictures in temporal electrode sites by means of one sample $t$ tests against zero.

A different picture emerged when analyzing the $\gamma-40$ band corrected for the neutral condition. A significant hemisphere by valence interaction $(F(1,8)=9.2, P<0.02)$ resulted from higher $\gamma$ - 40 power for positive valence over the right hemisphere and higher power for negative valence over the left hemisphere (Fig. 5). In general, $\gamma$-1 power was significantly higher on right hemisphere temporal electrodes as compared to the analogue electrodes over the left hemisphere $(F(1,8)=19.7, P<0.003)$.

When compared to the neutral condition, both, negative and positive valence induced significantly higher $\gamma-40$ power over both hemispheres as verified by one sample $t$ tests against zero (listed in Table 1).

No effects were found for $\gamma-60(50-70 \mathrm{~Hz})$ and $\gamma-80(70$ $90 \mathrm{~Hz}$ ) difference power. In particular, there was no indication for an interaction with valence.

\section{Discussion}

Perception, experience and expression of emotions may be represented in neuronal networks connecting subcortical (limbic) structures with neocortical areas (Tucker and Dawson, 1984; Derryberry and Tucker, 1992; Liotti and Tucker, 1995). This model postulates that widespread rather than focal neuronal activity is specifically related to emotional processing, a prediction that is consistent with the present experimental outcome. Processing of emotional pictures induces topographically specific cortical activity in EEG gamma from 30 to $50 \mathrm{~Hz}$. The valence hypothesis of hemispheric asymmetries predicts a significant valence by hemisphere interaction with a shift of relatively higher cortical activity for negative valence over the right hemisphere. Such an interaction was statistically pronounced but restricted to the $40 \mathrm{~Hz}$ band $(30-50 \mathrm{~Hz})$. However, we found exactly the opposite pattern with higher activity in the $30-50 \mathrm{~Hz}$ band over right anterior and posterior

Table 1

t tests against zero ${ }^{\mathrm{a}}$

\begin{tabular}{lll}
\hline & Left hemisphere & Right hemisphere \\
\hline Positive valance & $2.8^{*}$ & $5.1 * * *$ \\
Negative valance & $2.3^{* *}$ & $2.7^{*}$ \\
\hline
\end{tabular}

${ }^{a}$ d.f. $=8 . * P<0.05 ; * * P=0.05 ; * * * P<0.01$ 


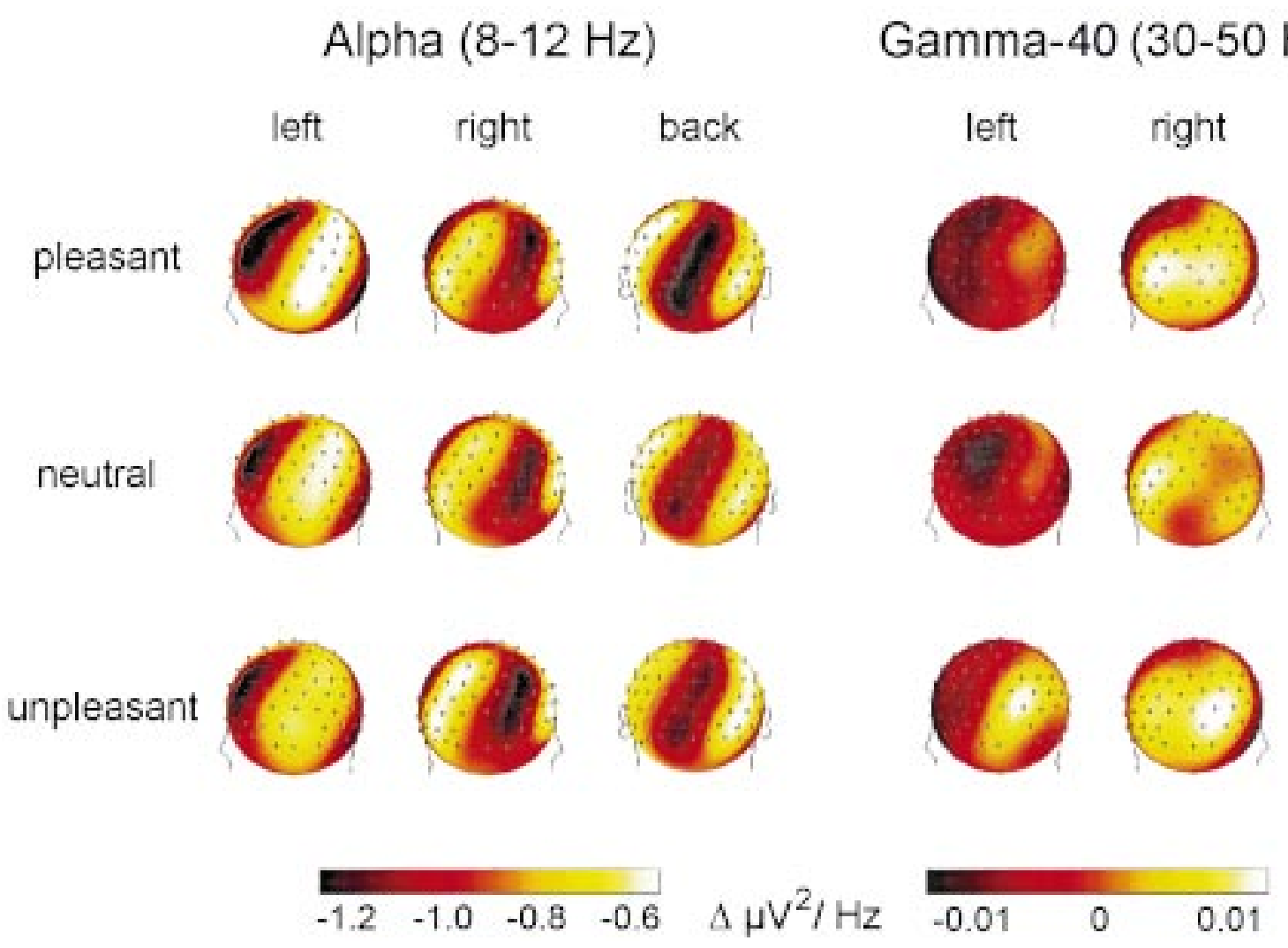

Fig. 4. Baseline corrected grand mean spherical spline topographical distributions of the alpha $(8-12 \mathrm{~Hz})$ and $\gamma-40(30-50 \mathrm{~Hz})$ frequency band for the 3 affective categories. Note: different scales for the alpha and gamma band.

temporal sites during the processing of pleasant pictures. In accordance with the general valence hypothesis, overall power was higher at right temporal and frontal sites than at left hemisphere electrodes. These findings are in line with observations in stroke patients, who exhibit more pronounced deficits with respect to both emotion perception

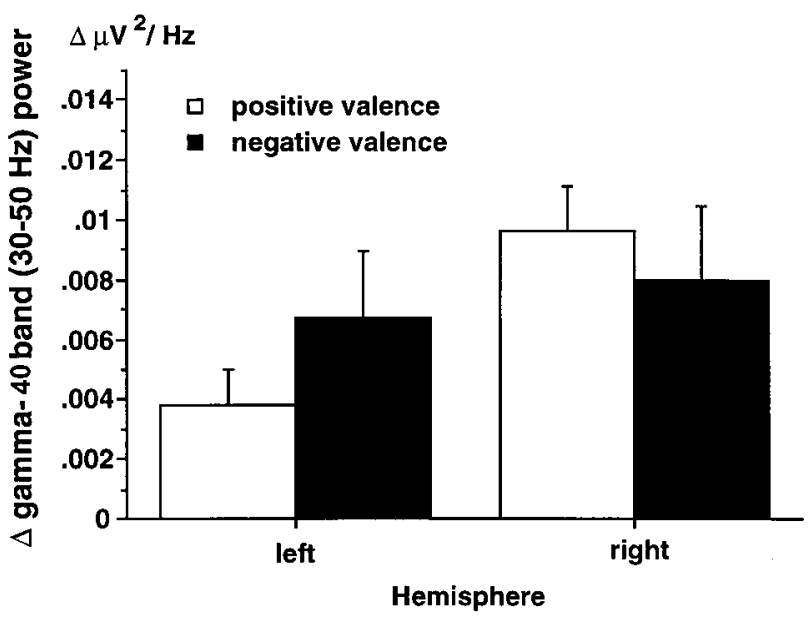

Fig. 5. Grand mean (+ standard errors) spectral power across temporal regional means for the left and right hemisphere across pleasant (white bars) and unpleasant pictures (black bars) with the neutral condition being subtracted. and expression after right hemisphere damage (see Borod, 1992, for an overview). The more specific assumption that the left hemisphere would produce more gamma activity when positive emotional material is to be processed, however, can clearly be rejected on the basis of the present results. The few studies which analyzed higher frequency components showed - in line with the present findings higher activity for positive valence at right hemisphere temporal electrodes (Tucker, 1981; Tucker and Dawson, 1984; Ray and Cole, 1985; Aftanas et al., 1998).

As discussed in the introduction, few studies exist which have analyzed the behavior in higher EEG frequency bands. Most reports limited their analysis to alpha desynchronization (Davidson, 1998). In the present study, we did not detect any sensitivity of alpha activity to the valence of emotional processing. Several factors may account for this. First, we have subtracted the mean activity phaselocked to picture onset prior to transformation into the frequency domain. The visual evoked response and the $\mathrm{P} 1 / \mathrm{N} 1$ complex, in particular, add to the power in the alpha band (Pantev et al., 1994) and thus may have contributed to the effects in previous reports. Second, contrary to other studies in the field, we have measured the emotional conditions in relation to a neutral baseline. It might be the case that alpha desynchronization effects appear when positive and negative valence are contrasted without correction 
for watching neutral pictures. Third, different configurations of electrode sites may produce different effects. In order not to violate statistical assumptions, we refrained from comparing every electrode location and any combination with its analogue of the opposite hemisphere.

As far as we know, the present study is the first to test cortical responses in the respective frequency bands by correcting for (a) general noise, which was operationalized by subtracting the respective power of a pre-stimulus time period, and, (b) calculating the absolute level of hemispheric function for negative and positive valence by subtracting the power while watching neutral slides. This procedure resulted in a significant increase only in $30-50 \mathrm{~Hz}$ band relative to the neutral condition across anterior and posterior temporal sites for the left and right hemisphere and for negative and positive valence. Using functional magnetic resonance imaging (fMRI), Lang and coworkers (1998) observed that emotional IAPS pictures were associated with enhanced metabolic activity in all sampled brain regions. Aside from activation of the right fusiform gyrus, and the right inferior and superior parietal lobules they reported bilateral activity in the occipital lobe. Using positron emission tomographic (PET) measurements, Lane and coworkers (Lane et al., 1997) demonstrated a selective increase of regional cerebral blood flow in the left parahippocampal gyrus, hippocampus and amygdala during viewing unpleasant IAPS pictures.

Posner and colleagues (Posner and Petersen, 1990; Posner and Dehaene, 1994) suggested a center in the anterior cingulate that may have an important role in priming the visual cortex for processing visual information. In addition, Davidson (1984), 1995) Davidson et al. (1990); Fox (1991) has emphasized the role of anterior cortical areas on emotion on the basis of observations of patients with anterior damages and the measure of alpha desynchronization. The present experiment could not replicate these findings of pronounced alpha desynchronization over left anterior regions for positive and right hemisphere anterior alpha desynchronization for negative valence.

Contamination of muscle activity must be a major concern when analyzing the EEG in the gamma-band range. Muscle activity shows its peak amplitude well beyond $60 \mathrm{~Hz}$ (Cacioppo et al., 1990; Pulvermüller et al., 1997), which is above the $30-50 \mathrm{~Hz}$ band whose experimental results were most pronounced. In addition, it is unlikely that the subjects would systematically tense right head muscles when pleasant pictures were presented and left head muscles while watching unpleasant pictures. The topographic distribution presently observed is also not consistent with muscle tensioning.

In sum, the present results suggest the activity of a wide spread cortical network predominantly in right frontal and temporal and to a lesser degree in left temporal areas. Thus, the present results support the idea of the existence of asymmetric cortico-limbic networks (Liotti and Tucker, 1995) and the gamma band topography of the present study suggests a contribution of the ventral visual pathway in combination with anterior areas. Whether presenting visual emotional material or imagination results in a different topography of gamma band activity requires further research. As with pictures, subjects should also be instructed to imagine a neutral scene in order to control for effects of imagination. The present study, however, can be seen as a first step to uncover a possible relation between gamma band activity and emotion.

\section{Acknowledgements}

We are grateful to Ursula Lommen and Klaus Lang for help in data acquisition and to Lisa Green for editorial support. Research was supported by grants from the Deutsche Forschungsgemeinschaft and the Human Frontier Science Program.

\section{Appendix A. IAPS numbers}

The IAPS numbers of the pictures used in the present study.

Pleasant pictures: 2050, 2070, 2080, 2160, 2165, 2170, 2311, 2340, 2341, 2360, 4650, 4651, 4652, 4658, 4659, 4660, 4664, 4670, 4680, 4690.

Neutral pictures: $2190,2200,221,0$ 2230, 2381, 2440, 2480, 2570, 2850, 7002, 7009, 7010, 7020, 7030, 7040, 7080, 7175, 7233, 7235, 9070 .

Unpleasant pictures: 1050, 1120, 1201, 1300, 1930, 3000, $3010,3050,3060,3071,3080,3102,3110,3130,3530$, $6260,6350,6510,6540,9405$.

\section{References}

Aftanas LI, Lotova NV, Koshkarov VI, Makhnev VP, Mordvintsev YN, Popov SA. Non-linear dynamic complexity of the human EEG during evoked emotions. Int J Psychophysiol 1998;28:63-76.

Borod JC. Interhemispheric and intrahemispheric control of emotion: a focus on unilateral brain damage. $\mathrm{J}$ Consult Clin Psychol 1992;60:339-348.

Bradley MM, Lang PJ. Measuring emotion: the self-assessment manikin and the semantic differential. J Behav Therapy Exp Psychiatry 1994;25:49-59.

Cacioppo JT, Tassinary LG, Fridlund AJ. The skeletomotor system, Cambridge: Cambridge University Press, 1990. pp. 325-384.

Davidson RJ. Affect, cognition and hemispheric specialization. In: Izard CE, Kagan J, Zajonc R, editors. Emotions, cognition, and behavior. Cambridge, England: Cambridge Press, 1984. pp. 320-365.

Davidson RJ. Cerebral asymmetry, emotion and affective style, Cambridge, MA: MIT Press, 1995. pp. 361-387.

Davidson RJ. Anterior electrophysiological asymmetries, emotion, and depression: conceptual and methodological conundrums. Psychophysiology 1998;35:607-614.

Davidson RJ, Hugdahl K. Brain Asymmetry, MIT Press: Cambridge, MA, 1995.

Davidson RJ, Ekman P, Saron CD, Senulis JA, Friesen WV. Approachwithdrawal and cerebral asymmetry: emotional expression and brain physiology. I. J Pers Soc Psychol 1990;58:330-341. 
Derryberry D, Tucker DM. Neural mechanisms of emotion. J Consult Clin Psychol 1992;60:329-338.

Eckhorn R, Reitboeck HJ, Arndt M, Dicke P. Feature linking via synchronization among distributed assemblies: simulations of results from cat visual cortex. Neural Computation 1990;2:293-307.

Ehrlichman H. Hemispheric asymmetry and positive-negative affect. In: Ottoson D, editor. Duality and unity of the brain, Hempshire: Macmillan, 1987. pp. 194-206.

Feige B. Oscillatory brain activity and its analysis on the basis of MEG and EEG. Münster: Westfälische Wilhelmsuniversität Münster 1996;1.

Fox NA. If it's not left, it's right. Electroencephalograph asymmetry and the development of emotion. Am Psychol 1991;46:863-872.

Gruber T, Müller MM, Keil A, Elbert T. Selective visual-spatial attention alters induced gamma band responses in the human EEG. Clin Neurophysiol 1999; in press.

Hellige JB. Hemispheric asymmetry, Cambridge: Harvard Univ Press, 1993.

Hirschman RS, Safer MA. Hemisphere differences in perceiving positive and negative emotions. Cortex 1982;18:569-580.

Keil A, Müller MM, Ray WJ, Elbert T, Gruber T. Does gamma band activity reflect perception? J Neurosci 1999; in press.

Lane RD, Reiman EM, Bradley MM, Lang PJ, Ahern GL, Davidson RJ, Schwartz GE. Neuroanatomical correlates of pleasant and unpleasant emotion. Neuropsychologia 1997;35:1437-1444.

Lang MM, Cuthbert BN. International affective picture system (IAPS): Technical manual and affective ratings. Gainesville: University of Florida. The Center for Research in Psychophysiology, 1997.

Lang PJ, Bradley MM, Fitzsimmons JR, Cuthbert BN, Scott JD, Moulder B, Nangia V. Emotional arousal and activation of the visual cortex: an fMRI analysis. Psychophysiology 1998;35:199-210.

LeDoux JE. In search of an emotional system in the brain: leaping from fear to emotion and consciousness. In: Gazzaniga MS, editor. Cognitive Neurosciences, Cambridge: MIT Press, 1995. pp. 1049-1061.

Liotti M, Tucker DM. Emotion in asymmetric corticolimbic networks. In: Davidson RJ, Hugdahl K, editors. Brain asymmetry, Cambridge: MIT Press, 1995. pp. 389-423.

Makeig S. Auditory event-related dynamics of the EEG spectrum and effects of exposure to tones. Electroenceph clin Neurophysiol 1993;86:283-293.

Müller, MM, Oscillatory cortical activities in the human brain.Universität Konstanz, Habilitation Thesis, Konstanz. 1998.

Müller MM, Bosch J, Elbert T, Kreiter A. Valdes Sosa. M., Valdes Sosa, P., Rockstroh, B., Visually induced gamma-band responses in human elec- troencephalographic activity - a link to animal studies. Exp Brain Res 1996;112:96-102.

Müller MM, Junghöfer M, Elbert T, Rockstroh B. Visually induced gamma-band responses to coherent and incoherent motion: a replication study. NeuroReport 1997;8:2575-2579.

Pantev C, Elbert T, Lütkenhöner B. Oscillatory event-related brain dynamics, New York: Plenum Press, 1994.

Perrin F, Pernier J, Bertrand O, Echallier JF. Spherical splines for scalp potential and current source density mapping. Electroenceph clin Neurophysiol 1989;72:184-187.

Posner MI, Dehaene S. Attentional networks. Trends Neurosci 1994;17:7579.

Posner IP, Petersen SE. The attention system of the human brain. Ann Rev Neurosci 1990;13:25-42.

Pulvermüller F, Lutzenberger W, Preissl H, Birbaumer N. Spectral responses in the gamma-band: Physiological signs of higher cognitive processes. NeuroReport 1995;6:2059-2064.

Pulvermüller F, Birbaumer N, Lutzenberger W, Mohr B. High-frequency brain activity: its possible role in attention, perception and language processing. Prog Neurobiol 1997;52:427-444.

Ray WJ, Cole HW. EEG alpha activity reflects attentional demands, and beta activity reflects emotional and cognitive processes. Science 1985;228:750-752.

Silberman EK, Weingartner H. Hemispheric lateralization of functions related to emotion. Brain Cog 1986;5:322-353.

Singer W, Gray CM. Visual feature integration and the temporal correlation hypothesis. Ann Rev Neurosci 1995;18:555-586.

Tallon C, Bertrand O, Bouchet P, Pernier J. Gamma-range activity evoked by coherent visual stimuli in humans. Eur J Neurosci 1995;7:12851291.

Tallon-Baudry C, Bertrand O, Delpuech C, Pernier J. Oscillatory gammaband $(30-70 \mathrm{~Hz})$ activity induced by a visual search task in human. J Neurosci 1997a;17:722-734.

Tallon-Baudry C, Bertrand O, Wienbruch C, Ross B, Pantev C. Combined EEG and MEG recordings of visual 40-Hz responses to illusory triangles in human. NeuroReport 1997b;8:1103-1107.

Tallon-Baudry C, Bertrand O, Peronnet F, Pernier J. Induced gamma-band activity during the delay of a visual short-term memory task in humans. J Neurosci 1998;18:4244-4254.

Tucker DM. Lateral brain function, emotion and conceptualization. Psychological Bulletin 1981;89:19-46.

Tucker DM, Dawson SL. Asymmetric EEG changes as method actors generated emotions. Biol Psychol 1984;19:63-75. 